Psychotherapeut 2021 66:195-202 https://doi.org/10.1007/s00278-020-00484-0 Angenommen: 8. Dezember 2020 Online publiziert: 13. Januar 2021 (c) Springer Medizin Verlag $\mathrm{GmbH}$, ein Teil von Springer Nature 2021, korrigierte Publikation 2021

Die Onlinepsychotherapie hat in Zeiten der Coronapandemie eine Trendwende erfahren und dies nicht nur hinsichtlich der gesetzlichen Regelungen, sondern auch bezüglich der Inanspruchnahme durch Psychotherapeuten. Studien vor der Pandemie zeigten, dass die Offenheit für E-Mental-Health-Anwendungen von der Therapieschule (Vigerland et al. 2014) sowie von Technikaffinität und -erfahrung (Eichenberg et al. 2016a; Kerst et al. 2019) des Therapeuten abhängig ist. In pandemischen Krisensituationen scheinen sich diese Faktoren aufzulösen, da der Großteil der Therapeuten ad hoc auf das video(telefonie)basierte Behandlungssetting umgestiegen ist.

\section{Zur Notwendigkeit der psychotherapeutischen Onlineversorgung}

Psychotherapeutische Versorgungsangebote auch unter den Bedingungen und Belastungen der aktuellen Coronapandemie offen zu halten, ist wichtiger denn je. Das heißt, benötigt werden psychotherapeutische Settings, die auch in Zeiten von Lockdowns, in Zeiten von Angst vor sozialkörperlichem Kontakt aufgrund möglicher Ansteckung diejenigen unterstützen, die vor der pandemischen Krise schon psychisch belastet waren oder in der Situation Belastungsreaktionen entwickelt haben. Ein aktuelles Review zeigt, dass z. B. unter Quarantänebedingungen nicht nur die allgemeine psychosoziale Belastung zunimmt, sondern insbesondere Tätige im Gesundheitswesen eine vulnerable Gruppe darstellen (Röhr et al. 2020). Dass es absolut erforderlich ist,

Christiane Eichenberg

Institut für Psychosomatik, Fakultät für Medizin, Sigmund Freud PrivatUniversität Wien, Wien, Österreich

\title{
Onlinepsychotherapie in Zeiten der Coronapandemie
}

die notwendigen Versorgungsstrukturen $\mathrm{zu}$ ermöglichen, verdeutlichen weitere Studien, die die Prävalenz psychischer Erkrankungen während der aktuellen Coronakrise erfasst haben. So zeigt zum einen eine Studienserie der DonauUniversität Krems, dass Psychotherapiepatienten u.a. aufgrund der gesetzlich angeordneten Präventionsmaßnahmen eine Aggravation ihrer Symptomatik erfahren haben. Befragt wurden über 1500 österreichische Psychotherapeuten, die bei $70 \%$ ihrer Patienten negative Auswirkungen in dem Sinne wahrnahmen, dass bestehende Symptome sich verschlimmerten und bereits überwundene Traumata reaktiviert wurden (https:// news.orf.at/stories/3163161. Zugegriffen: 01.11.2020). Zum anderen hat eine Studie die Zunahme klinisch relevanter Störungen in der Phase des Lockdowns erhoben. An einer für Österreich repräsentativen Stichprobe von 1009 Menschen konnte festgestellt werden, dass die Prävalenz depressiver Symptome von etwa $4 \%$ auf über $20 \%$ angestiegen ist. Eine ähnlich starke Zunahme findet sich bei Angstsymptomen, die sich von 5\% auf $19 \%$ erhöhten (Pieh et al. 2020). Studien aus anderen Ländern kommen $\mathrm{zu}$ ähnlichen Ergebnissen: Im United Kingdom (Pierce et al. 2020) wurden Probanden aus der Allgemeinbevölkerung $(n=17.452)$ mithilfe des General Health Questionnaire (GHQ-12; Goldberg und Williams 1988) vor und nach dem Lockdown untersucht. Das heißt, es wurden die Items des GHQ-12 im April 2020, einen Monat nach dem Lockdown, erfasst und die Ergebnisse mit den Befunden einer nationalen, longitudinalen Kohortenstudie aus dem Jahr 2018/2019 verglichen. Demnach stieg die Prävalenz psychischer Störungen in der Bevöl- kerung von $18,9 \%$ im Jahr 2018/2019 auf 27,3\% im April 2020. Der mittlere GHQ-12-Wert stieg in diesem Zeitraum ebenfalls an (von 11,5\% auf 12,6\%). Der Anstieg war bei den Jüngeren (18-34 J.), bei Frauen sowie bei Menschen, die mit kleinen Kindern leben, am größten. In einer eigenen Studie führten die Autoren eine nichtrepräsentative Onlinebefragung an 3003 in Deutschland und Österreich lebenden Personen durch, mit dem Ziel, die psychischen Belastungen durch die gesetzlich angeordneten Präventionsmaßnahmen in der Situation des Lockdowns zu erfassen (ausführlich: Eichenberg et al. im Druck a, b). Auch hier wurden verschiedene Gruppen mit unterschiedlichem Belastungs- und Ressourcenprofilen identifiziert. Drei von 4 dieser Gruppen wiesen Konstellationen auf, die einen psychosozialen Unterstützungsbedarf nahelegen, um einerseits Belastungen $\mathrm{zu}$ reduzieren, andererseits aber auch, um die z.T. mangelnde Compliance in Bezug auf die gesetzlich angeordneten Präventionsmaßnahmen im Sinne eines gesamtgesellschaftlichen Schutzes zu fördern.

\section{Varianten von Online- psychotherapie}

Wird die Onlinepsychotherapie in Zeiten der Coronapandemie in den Blick genommen, ist zunächst zwischen ihren verschiedenen möglichen Varianten $\mathrm{zu}$ unterscheiden.

Auch wenn das Spektrum von E-Mental-Health, d. h. die digitale Medienunterstützung in der Prävention, Behandlung und Rehabilitation psychischer Störungen, sehr breit ist und weit mehr Optionen umfasst als die Onlinetherapie (zur Übersicht: Eichenberg und Kühne 2014), 
sind nur wenige digitale Szenarien für die ambulante Psychotherapie in der Coronakrise unmittelbar relevant.

\section{Reine Onlinetherapie}

Bei reinen Onlinetherapien handelt es sich zum einen um selbstgesteuerte Interventionsprogramme, die auf einem festgelegten Behandlungsprotokoll basieren. Dabei gibt es verschiedene Stufen dieser Programme, die von selbstgesteuerten Onlinetrainings zur Selbsthilfe (sog. internetbasierte ungeleitete Selbsthilfe- oder Interventionsangebote) bis hin zur Onlinepsychotherapie mit von Patienten $\mathrm{zu}$ bearbeitenden Modulen reichen, in die oftmals ergänzende Kontakte mit einem Psychotherapeuten, z.B. per E-Mail, Telefon oder Kurznachrichtendienst, integriert werden (sog. internetbasierte geleitete Selbsthilfe- oder Interventionsangebote). Daher handelt es sich in den meisten Fällen um „Minimal-contact"-Angebote, da sich diese Konzeption therapeutisch als am effektivsten erwiesen hat. Die meisten dieser Angebote sind kognitivbehavioral orientiert, mit inzwischen solider empirischer Evidenz (Andersson et al. 2014; Peñate und Fumero 2016) für v.a. depressive Erkrankungen und Angststörungen (Stein et al. 2018). Allerdings gibt es derweil entsprechende Angebote zu fast allen Problem- und Störungsbereichen.

Inzwischen existieren auch positiv evaluierte Angebote, die auf psychodynamischer Grundlage beruhen und sich an Einzelpersonen wenden (z. B. „KENOnline“; Zwerenz et al. 2017) oder im Gruppensetting durchgeführt werden (Lemma und Fonagy 2013).

Zum anderen können Onlinetherapien auch als „Remote“-Therapien geführt werden, d.h., die Sitzungen finden ausschließlich per Telefon oder-inzwischen zunehmend häufiger - per Videokonferenz statt, was auch in psychodynamischen Therapien im Bereich digitaler Angebote seit Längerem eingesetzt wird (Eichenberg und Hübner 2018). In Zeiten von Corona bedeutet dies, dass Psychotherapeuten neue Patienten aufnehmen und diese entweder per Telefon oder im Videosetting behandeln.

\section{"Blended therapy"}

Bei den sog. Blended-therapy-Ansätzen wird die traditionelle Behandlung mit digitalen Interventionen oder werden verschiedene digitale Behandlungsmodule, wie z.B. internetbasierte Interventionsprogramme plus videobasierte Psychotherapie, miteinander kombiniert (z. B. Etzelmüller et al. 2018). Auch hier sind verschiedene Anwendungsmodi $\mathrm{zu}$ unterscheiden, d.h., in verschiedene Behandlungsphasen können digitale Angebote auf unterschiedliche Weise integriert werden. Beispielhaft zu nennen sind die beiden Programme der Ambulanz der Psychosomatischen Klinik Kloster Dießen „Online-Ambulanz-Service für Internetsüchtige“ (OASIS; https:// www.onlinesucht-ambulanz.de. Zugegriffen:01.11.2020) und „Onlinebasiertes Motivationsprogramm zur Reduktion des problematischen Medienkonsums und Förderung der Behandlungsmotivation bei Menschen mit Computerspielabhängigkeit und Internetsucht " (OMPRIS; https://www.onlinesucht-hilfe.com. Zugegriffen: 01.11.2020). Diese bieten von exzessiver Internetnutzung Betroffenen videobasierte Sprechstunden an, um sowohl die Behandlungsmotivation vor der Therapie zu stärken, als auch die Betroffenen an geeignete Therapieangebote vor Ort zu vermitteln. Auch in der Nachsorge können z.B. digitale poststationäre Programme die Therapieeffekte nachhaltig sichern helfen (z. B. Wolf et al. 2008).

Während der Behandlung können z.B. die oben genannten ungeleiteten und geleiteten Selbsthilfeprogramme integriert oder aber traditionelle Sitzungen in der Therapiepraxis mit digitalen Sitzungen abgewechselt werden. In den allermeisten Fällen werden Psychotherapeuten in der Coronakrise ihre Patienten in laufenden Behandlungen nur per (Video-)Telefonie weiterbehandeln.

Während umfangreiche Literatur über die entscheidende Rolle der therapeutischen Beziehung in der "Face-to-face“Psychotherapie existiert, ist die Forschung über die therapeutische Allianz bei Onlinetherapien noch begrenzt. Belege dafür, dass im Rahmen einer Onlinepsychotherapie mit Kontakt zu einem Therapeuten eine stabile und positive therapeutische Beziehung aufgebaut werden kann, stehen jedoch schon lange zur Verfügung (für eine Übersicht zu den existierenden Studien zur therapeutischen Beziehung im Onlinesetting, differenziert nach kognitivbehavioralen und psychodynamischen Onlinetherapien: Eichenberg und Hübner 2020). Weniger Befunde gibt es zum spezifischen videobasierten Setting. In einem systematischen Review über Videokonferenzpsychotherapie wurden 7 "randomized controlled trials" (RCT), 3 fallbezogene Studien und mehrere unkontrollierte Pilot- oder Einzelfallstudien identifiziert, die die therapeutische Allianz gemessen haben (Simpson und Reid 2014). Insgesamt war die Allianz bei Videokonferenztherapien homogen hoch und entsprach der von Face-toface-Therapien. Zum gleichen Ergebnis kommt auch eine aktuelle Studie, die die Einschätzungen von Patienten hinsichtlich der therapeutischen Allianz im Videotherapiesetting mithilfe von Interviews erhoben hat (Etzelmüller et al. 2018).

Allerdings ist bisher auf empirischer Basis noch gar nichts darüber bekannt, wie Therapeut und Patient diese Settingwechsel erleben, d.h. den Wechsel aus dem bekannten Setting in der Therapiepraxis auf das Onlinesetting und auch wieder zurück, wenn - beispielsweise nach Lockdowns - Behandlungen wieder im traditionellen Setting möglich sind. Entsprechende Begleitforschung ist wichtig, um die Auswirkungen dieser Wechsel auf die therapeutische Beziehung sowohl therapeutenseits als auch patientenseits zu erfassen. Es wurde an der Sigmund Freud PrivatUniversität Berlin (Fakultät für Psychotherapiewissenschaft) eine entsprechende Studie durchgeführt, deren Ergebnisse in Psychotherapeut, Schwerpunktheft „Digitale Psychotherapie“ (Sept. 2021) vorgestellt werden.

\section{Blended therapy in der Kinder- und Ju-} gendlichenpsychotherapie. Noch sehr viel dünner sind empirische Befunde zum Blended-Setting in der Behandlung von Kindern und Jugendlichen. Befragungen unter Psychotherapeuten 
Psychotherapeut 2021 ·66:195-202 https://doi.org/10.1007/s00278-020-00484-0

(c) Springer Medizin Verlag GmbH, ein Teil von Springer Nature 2021

\section{Eichenberg}

\section{Onlinepsychotherapie in Zeiten der Coronapandemie}

\section{Zusammenfassung}

Hintergrund. Die Prävalenz psychischer Erkrankungen in der Bevölkerung ist während der Coronapandemie aufgrund der mit ihr einhergehenden Belastungen enorm gestiegen. Umso wichtiger ist es, das psychotherapeutische Versorgungsangebot offen zu halten, auch in Zeiten, in denen notwendig ist, den sozialkörperlichen Kontakt einzuschränken. Damit wurden Psychotherapeuten ad hoc damit konfrontiert, ihr traditionelles Behandlungssetting zu erweitern und Fernhandlungen anzubieten. Fragestellung. Welche digitalen Behandlungsoptionen stehen Psychotherapeuten - auch vor dem Hintergrund gesetzlicher Regelungen - seit der Pandemie zur Verfügung? In welchem Ausmaß wurde die Videobzw. telefonbasierte Psychotherapie genutzt?
Welche Erfahrungen haben Psychotherapeuten mit digitalen Settings gemacht? Und wie ist die aktuelle Studienlage hinsichtlich der Effektivität und therapeutischen Allianz im Video(telefonie)-Setting zu beurteilen? Wie kann es gelingen, die eigene therapeutische Methode auf dieses neue Setting bei verschiedenen Patientengruppen (Kinder, Jugendliche, Erwachsene) anzupassen? Methoden. Diese Fragen werden anhand einer Literaturrecherche sowie klinischer Überlegungen beantwortet.

Ergebnisse. Ein Großteil der Psychotherapeuten hat sich sehr schnell auf Fernbehandlungen eingestellt, was der Gesetzgeber durch entsprechende Lockerungen bestehender Regelungen unterstützt hat, um in der pandemischen Krise sowohl bereits in Psychotherapie befindliche Patienten als auch neue Patienten ohne physischen Kontakt weiterbehandeln zu können. Es zeigte sich in Befragungsstudien, dass Psychotherapeuten die Wirksamkeit dieses neuen Settings geringer einschätzen als das traditionelle Setting.

Diskussion. Fundierte Fortbildungen sind notwendig, um Psychotherapeuten in Theorie, Praxis und Selbsterfahrung für ein qualitätssicherndes Arbeiten im Video(telefonie)-Setting zu schulen.

Schlüsselwörter

E-Mental-Health · Fernbehandlung ·

Soziale Distanz - Therapeutische Allianz . Weiterbildung

\section{Online psychotherapy in times of the corona pandemic}

\section{Abstract}

Background. The prevalence of mental illnesses in the population has enormously increased during the corona pandemic due to the accompanying burdens and distress. Therefore, it is important to continue with the provision of psychotherapeutic treatment even in times of social distancing. As a result, psychotherapists were soon confronted with the need to expand their traditional treatment setting and offer remote treatment.

Objective. Which digital treatment options are available to psychotherapists since the pandemic, also with respect to the legal regulations? To what extent and how did they use video-based or telephone-based psychotherapy? What is the current stage of research regarding the effectiveness of video(telephony)? How can therapeutic alliance be built into video(telephony) settings? How can psychotherapists successfully apply their personal therapeutic method to different patient groups (children, adolescents, adults) in the new digital setting?

Methods. These questions are answered based on a literature search and clinical considerations.

Results. The majority of psychotherapists have immediately adapted to remote treatment. This has been supported by the government by expanding existing regulations in order to continue treatment with current and new patients without physical contact during the pandemic crisis. Survey studies have shown that psychotherapists consider the effectiveness of digital treatment settings to be lower than the traditional setting. Conclusion. Further training is necessary to educate psychotherapists in the theory, practice and self-awareness for quality assurance in the video(telephony) setting.

Keywords

E-mental health · Remote treatment · Social distance $\cdot$ Therapeutic alliance $\cdot$ Further education
(Deutsche Psychotherapeutenvereinigung 2020; s. unten) zeigten, dass bei dieser Altersgruppe die größten Bedenken bestehen, und zwar in dem Sinne, dass v.a. bei Kindern Videobehandlungen gar nicht möglich seien. Sindelar (2020) argumentiert jedoch, dass sich auch in der Kinder- und Jugendlichenpsychotherapie nicht mehr die Gretchenfrage stelle, ob Onlinetherapie möglich ist oder nicht, da dieses Setting z. B. während des Lockdowns die einzige Behandlungsmöglichkeit war. Vielmehr sei die Frage zu stellen, wie die Sprache des Spiels im Onlinesetting weitergeführt werden kann, was v. a. bei jüngeren Kindern zentral ist, da sie ihr inneres Erleben noch nicht verbalisieren können. So ist z. B. das gemeinsame Durchführen von Onlinespielen, eingebettet in eine Videokonferenz, eine mögliche Option, da die Auswahl der Spielfigur, die Art des Zusammen- oder Gegeneinanderspielens dem Psychotherapeuten die gleichen Interventionsmöglichkeiten durch therapeutische Techniken des Spiegelns, des Verbalisierens, des Markierens von Gefühlen, Erprobens von
Handlungsspielräumen wie im realen Spiel bietet. Voraussetzung ist natürlich, dass den Therapeuten die Chancen des „online gaming“ bekannt sind (Eichenberg et al. 2016b), ebenso wie eine weitere Form des Adjuvans von Psychotherapie, nämlich der Einbezug von „serious games“. Serious games sind Computerspiele, die neben dem Unterhaltungsaspekt das Ziel verfolgen, Wissen zu vermitteln bzw. Verhaltensänderungen zu unterstützen. Gerade für Kinder und Jugendliche existieren im psychotherapeutischen Bereich bereits gut evaluierte 
Spiele, die meist störungsbezogen sind (z.B. SPARX [,smart, positive, active, realistic, $X$-factor thoughts"] zu Prävention und Behandlung depressiver Störungen: https://www.sparx.org.nz. Zugegriffen: 01.11.2020; oder CampCope-A-Lot für Kinder mit Angststörungen: https://www.copingcatparents. com. Zugegriffen: 01.11.2020). Zwar sind manche Spiele (z.B. SPARX) so konzipiert, dass sie unabhängig von einer Psychotherapie genutzt werden, in der Regel werden sie jedoch in eine therapeutische Gesamtkonzeption integriert (für ein systematisches Review zur Effektivität von therapeutischen Serious games: Eichenberg und Schott 2017).

Sindelar (2020) betont, dass eine videobasierte Therapie für kleinere Kinder meist nicht möglich sei, in deren Behandlung aber die Elternarbeit vorrangig ist, die wiederum sehr gut im Videosetting umgesetzt werden kann. Dennoch kann das Videosetting auch kleinen Kindern die Möglichkeit bieten, die Beziehung zum Therapeuten zu halten, da eine - z. B. Lockdown-bedingte Unterbrechung - eine zusätzliche Belastung darstellen würde. Dass auch Kindern psychosoziale Unterstützungsoptionen geschaffen bzw. offengehalten werden müssen, zeigen Zahlen bezüglich ihrer Belastungen während der Coronapandemie: Eine Befragung der Universitätsklinik Hamburg-Eppendorf (https://www.uke.de/allgemein/presse/ pressemitteilungen/detailseite_96962.

html. Zugegriffen: 01.11.2020) ergab, dass die Lebensqualität der Kinder und Jugendlichen in Deutschland in der Coronapandemie deutlich gelitten hat. Die meisten Kinder und Jugendlichen (71\%) fühlten sich belastet, machten sich vermehrt Sorgen, achteten weniger auf ihre Gesundheit und beklagten häufiger Streit in der Familie. Genauso wie bei den Erwachsenen müssen aber auch bei dieser Gruppe differenzierte Reaktionen berücksichtigt werden. So fanden manche Kinder in dieser Krise auch Entlastung. In einer Onlineumfrage, an der 533 Eltern mit an Aufmerksamkeitsdefizit-/ Hyperaktivitätsstörung (ADHS) erkrankten Kindern in Frankreich (Bobo et al. 2020) teilnahmen, zeigte sich, dass die meisten Kinder nach Angaben ihrer
Eltern eine Stabilität oder Verbesserung ihres Wohlbefindens erlebten. Als Gründe gaben die Eltern eine Linderung schulbedingter Ängste, die flexible Anpassung an die Rhythmen der Kinder und ihr gesteigertes Bewusstsein für die Schwierigkeiten ihrer Kinder an.

\section{Gesetzliche Rahmenbedin- gungen zur Durchführung von Onlinepsychotherapie vor und seit der Coronapandemie}

\section{Situation in Deutschland}

Die digitale Medienunterstützung in der Psychotherapie wurde innerhalb des Berufsstands der Psychotherapeuten von Beginn an sehr kontrovers diskutiert. Während bis vor Kurzem Befürworter und Gegner heftig debattierten, ob Psychotherapie im Onlinesetting überhaupt lege artis sei, und ob in diesem Rahmen grundsätzlich eine therapeutische Beziehung aufgebaut werde könne, entwickelte sich im letzten Jahr - aufgrund einer soliden empirischen Basis - auch gesetzlich eine Auflockerung des traditionellen Face-to-face-Settings. Fachärzten bestimmter Fachgebiete war es schon seit dem 01.04.2017 möglich, Videosprechstunden anzubieten. Zum 01.04.2019 wurde die Videosprechstunde für alle Indikationen geöffnet. Damit wurde es auch möglich, dass Psychotherapeuten die Videosprechstunde abrechnen können, allerdings nur für maximal $20 \%$ ihrer Patienten. Seit dem 01.10.2019 zahlen die gesetzlichen Krankenkassen Ärzten und Psychotherapeuten, die Videosprechstunden durchführen, eine Anschubfinanzierung (https://www.kbv. de/html/1150_39923.php. Zugegriffen: 01.11.2020).

Die aktuelle Coronakrise hat sehr schnell eine absolute Trendwende im Bereich der Onlinepsychotherapie herbeigeführt. So haben die Kassenärztliche Bundesvereinigung und der GKV-Spitzenverband am 25.03.2020 beschlossen, dass eine psychotherapeutische Sprechstunde und probatorische Gespräche während der Coronapandemie auch per Videotelefonat möglich sind. Danach können in begründeten Einzelfällen die Beratung und Diagnostik von Patienten ohne unmittelbaren Kontakt erfolgen, d.h., es können auch neue Behandlungen mit Patienten, die nie in der psychotherapeutischen Praxis vor Ort gesehen wurden, initiiert werden.

Argumentiert wird, dass insbesondere Quarantänepatienten nicht anders $\mathrm{zu}$ versorgen seien, aber auch älteren Menschen nicht zumutbar sei, das Risiko einer Ansteckung auf dem Weg zur Praxis einzugehen. Auch für Gruppentherapien wurden Erleichterungen geschaffen. Bereits genehmigte Gruppentherapiesitzungen können unbürokratisch als Einzeltherapiesitzungen durchgeführt werden. Hierfür ist nur eine formlose Anzeige bei der Krankenkasse erforderlich. Zudem sind Videobehandlungen unbegrenzt möglich, d.h., ihre Beschränkung auf maximal $20 \%$ der Patienten ist während der Coronapandemie ausgesetzt. Dabei sind Telefonbehandlungen ebenfalls möglich, um auch jene Patienten versorgen $\mathrm{zu}$ können, die nicht über die technischen Voraussetzungen für eine Videotelefonie verfügen oder keine technische Kompetenzen dafür haben. In akuten Krisensituationen ist die Behandlung in der Therapiepraxis jedoch zwingend, ansonsten bleibt es dem Therapeuten freigestellt, ob er weiterhin Sitzungen (auch Gruppensitzungen) in seiner Praxis anbieten möchte (z. B. Psychotherapeutenkammer Berlin: https://www. psychotherapeutenkammer-berlin.de/ nachrichten/wichtige-informationenfuer-kammermitglieder-zu-den-auswir kungen-der-corona-krise. Zugegriffen: 01.11.2020).

Allerdings wurden diese gesetzlichen Lockerungen bezüglich der Videotherapie zunächst zeitlich befristet und zwar bis zum 30.09.2020. In einer Aktualisierung der Praxis-Info der Bundespsychotherapeutenkammer (BPTK) vom 09.10.2020 wird erläutert, dass diese Befristung nun bis zum 31.12.2020 verlängert wurde (https://www.bptk. de/wp-content/uploads/2020/06/BPtKPraxis-Info-Coronavirus.pdf. Zugegriffen: 01.11.2020).

Wichtig zu beachten ist, dass Messenger-Dienste nicht zulässig sind, sondern nur zertifizierte Videotelefonieprogramme (zur Übersicht: https:// 
www.krankenkassenzentrale.de/liste/ videosprechstunden. Zugegriffen: 01.11. 2020).

\section{Situation in der Schweiz}

In der deutschsprachigen Schweiz ist die Situation bezüglich der Onlinebehandlung schwierig. Die Föderation der Schweizer Psychologinnen und Psychologen fasst zusammen, dass die Berufsverbände die Korrektur eines entsprechenden Entscheids des Bundesamts für Gesundheit (BAG) fordern, da Onlinetherapien für alle zugänglich sein müssen. De facto werden Psychotherapien auf Distanz trotz des Lockdowns jedoch nur sehr beschränkt von der Krankenkasse bezahlt (https://www. psychologie.ch/online-therapie-mussfuer-alle-gleich-zugaenglich-sein. $\mathrm{Zu}$ gegriffen: 01.11.2020). Konkret bedeutet dies, dass das BAG im April 2020 zwar festlegte, dass Distanztherapien ohne Einschränkungen bis auf Weiteres auch von der Grundversicherung bezahlt werden. Das gilt allerdings nur dann, wenn sie von Psychiatern durchgeführt werden. Psychologische Psychotherapeuten, die in der Schweiz via delegierte Psychotherapie über die Grundversicherung abrechnen, dürfen nur $360 \mathrm{~min} /$ Quartal Distanztherapien über die Krankenkasse abrechnen. Der Bundesrat hat die Therapiedauer während der Coronazeit zwar von 240 auf 360 min erhöht, was aber natürlich nicht ausreichend ist, um Bestandspatienten weiterbehandeln $\mathrm{zu}$ können. Außerdem bedeutet es, dass Patienten, die sich von einem psychologischen Psychotherapeuten behandeln lassen und die ihre Therapie nicht selbst bezahlen können, massiv schlechter gestellt werden als Patienten, die bei einem Psychiater in Behandlung sind (zur ausführlichen Darstellung der komplexen Situation in der Schweiz wird auf den oben genannten Link verwiesen).

\section{Situation in Österreich}

Die Coronakrise hat hingegen in Österreich die Situation der Onlineversorgung drastisch verändert. Im Vergleich zu Deutschland waren die Änderungen noch bahnbrechender, da hier stärkere Restriktionen bestanden, d.h., die Onlinebehandlung in keinem Fall möglich war. In Österreich ist die psychotherapeutische von der klinisch-psychologischen Behandlung $\mathrm{zu}$ unterscheiden. Zum Beispiel gibt die „Internetrichtlinie für Psychotherapeutinnen und Psychotherapeuten - Kriterien zur Ausgestaltung der psychotherapeutischen Beratung via Internet" des Bundesministeriums für Soziales, Gesundheit, Pflege und Konsumentenschutz (https:// www.lasf.at/wp-content/uploads/2017/ 10/internetrichtlinie_03052012.pdf. Zugegriffen: 01.11.2020) an, dass Psychotherapie als Behandlung via Internet nicht lege artis sei. Dagegen übernehmen in Zeiten der „coronavirus disease 2019“ (COVID-19) die meisten Krankenkassen in Österreich erstmals auch Kosten für Psychotherapien via Telefon und Internet. Für die klinisch-psychologische Behandlung wurde die Kassenfinanzierung aufgrund des erhöhten Bedarfs ad hoc gefordert (https://zph.meduniwien.ac.

Hier steht eine Anzeige. 
at/news/single-view/?tx_ttnews\%5Btt_ news\%5D $=4700 \& \mathrm{cHash}=088 \mathrm{f}$ a $27 \mathrm{f0} 295$ 485a9148779b072c969. Zugegriffen: 01.11.2020).

Aufgrund des dynamischen Geschehens der COVID-19-Pandemie ist es jedoch notwendig, zeitliche Befristungen von Onlinetherapieoptionen nicht so eng $\mathrm{zu}$ setzen, sondern vielmehr zu ermöglichen, gesetzliche Lockerungen mittelbis längerfristig umzusetzen, damit Psychotherapeuten und Patienten die Sicherheit haben, hierauf länger zurückgreifen zu können. Andernfalls besteht das Risiko, die therapeutische Beziehung durch ständige Settingwechsel zu belasten. Es sollte dem therapeutischen Paar daher offenstehen, die während des Lockdowns notwendige Umstellung des Settings beibehalten zu dürfen, u. U. bis zum Ende der Therapie. Ebenso zu denken ist an Psychotherapeuten und Patienten, die auch in Phasen niedriger Infektionsraten das Onlinesetting präferieren, weil sie u. U. einer Risikogruppe angehören oder das Bedürfnis haben, sich auch in diesen Phasen maximal vor einer Ansteckung zu schützen.

\section{Erfahrungen von Psycho- therapeuten mit Onlinetherapie während der Coronapandemie}

Während in Zeiten des Lockdowns bereits erste Erfahrungsberichte von Psychotherapeuten veröffentlicht wurden, die aus ihrem Behandlungsalltag im Videotelefoniesetting berichten (https://www.bptk.de/der-schutzraumder-praxis-fehlt/. Zugegriffen: 01.11 . 2020), liegen inzwischen empirische Studien vor, die diese Erfahrungen systematisch erhoben haben.

In einer Umfrage der Deutschen Psychotherapeutenvereinigung (2020) zufolge, an der Anfang April 20204466 Psychotherapeuten teilnahmen, gaben $77 \%$ der Befragungsteilnehmer an, dass sie die Möglichkeiten der Videobehandlung nutzen, $95 \%$ davon erst seit Beginn der Coronakrise, d.h., sie hatten sich ad hoc auf diese neue Setting eingestellt. Ein weiteres Ergebnis war, dass sich die Therapeuten auf die Videobehandlung eingelassen haben, trotz behandlungstechnischer Bedenken, um bestimmte
Patientengruppen weiterversorgen $\mathrm{zu}$ können. Manche genannten Nachteile - wie z.B. Kinder nicht per Videosetting behandeln zu können - würden relativiert werden können, wenn entsprechende Weiterbildungen etabliert würden (s. die obigen Ausführungen zur Onlinebehandlung von Kindern und Jugendlichen nach Sindelar 2020).

Die medienunterstützten Settingoptionen im Bereich der psychotherapeutischen Versorgung wurden auch in Österreich stark genutzt. Dies zeigte eine Onlineumfrage unter 1500 österreichischen Psychotherapeuten: Die Behandlungszahlen via Telefon stiegen um $979 \%$ und via Internet um $1561 \%$ (https://www.donau-uni.ac.at/ de/aktuelles/news/2020/psychotherapieauf-distanz-waehrend-covid-19-0.html. Zugegriffen: 01.11.2020).

In einer ländervergleichenden Studie (Deutschland, Tschechien und Slowakei) mit insgesamt 338 befragten Psychotherapeuten (Humer et al. 2020) wurde deren Angst vor einer Infektion mit dem „severe acute respiratory syndrome coronavirus 2“ (SARS-CoV-2) während einer Face-to-face-Psychotherapie erhoben. Ferner wurde die Zahl der Patienten, die während der COVID-19-Situation sowie (rückblickend) in den Monaten zuvor durchschnittlich pro Woche (im persönlichen Kontakt, per Telefon, über das Internet) behandelt wurden, ermittelt. Die Angst vor einer SARS-CoV2-Infektion war in der Slowakei am höchsten und in Deutschland am niedrigsten; Frauen berichteten signifikant mehr Angst als Männer. In allen Ländern ging die Zahl der Patienten, die im Durchschnitt pro Woche im persönlichen Kontakt behandelt wurden, zurück, und die Fernbehandlungen nahmen zu, wobei mehr Patienten über das Internet als über das Telefon behandelt wurden. Darüber hinaus behandelten weibliche Psychotherapeutinnen weniger Patienten im persönlichen Kontakt, während sie mehr Patienten über das Telefon behandelten als ihre männlichen Kollegen. Insgesamt unterschied sich die Gesamtzahl der während der COVID19-Pandemie behandelten Patienten nicht von der Zahl der Patienten in den Monaten davor, was darauf hindeutet, dass das Angebot an psychosozialer Versorgung während COVID-19-Pandemie aufrechterhalten werden konnte.

Insgesamt ist beachtenswert, dass ein Großteil der Psychotherapeuten die Herausforderung angenommen hat, sich - trotz Bedenken - auf ein unbekanntes Setting eingelassen zu haben, und das, obwohl diese nicht nur als Behandler, sondern auch als Privatpersonen einen Ausnahmezustand erleben, d.h., auch selbst betroffen sind. Daher sind auch für Psychotherapeuten im Umgang mit der Coronapandemie Maßnahmen der Psychohygiene von zentraler Bedeutung (Zurek 2020).

Gleichzeitig scheinen die im Lockdown gewonnenen Erfahrungen eine Einstellungsänderung der Psychotherapeuten gegenüber Onlinetherapie herbeigeführt zu haben. Eine österreichische Befragung von 700 Psychotherapeuten zeigte, dass vor der COVID-19-Pandemie nur ca. ein Drittel gegenüber der Onlinetherapie positiv eingestellt war; nach den Erfahrungen während der Pandemie änderte sich dies auf zwei Drittel. In der Befragung wurde auch deutlich, dass die Psychotherapeuten für eine weitere Lockerung der gesetzlichen Bestimmungen plädierten, denn 9 von 10 Psychotherapeuten gaben an, sich vorstellen zu können, das Onlinesetting auch "nach Corona" flexibel anzuwenden, wenn es in den Leistungskatalog der Krankenkassen aufgenommen würde (https://www.sfu.ac.at/ wp-content/uploads/SFU_Presseinfos_ 2020_08_28_E-Psychotherapie.pdf. Zugegriffen: 01.11.2020).

\section{Weiterbildungen im Bereich der Onlinepsychotherapie}

Unbestritten ist auch in Fachkreisen, dass die Videobehandlung keine „Faceto-face"-Behandlung ist, sondern vielmehr nach Kühne und Hintenberger (2020) ein „Camera-to-camera“-Kontakt. Die Interaktion in diesem Setting weist Besonderheiten auf, die für das therapeutische Arbeiten besonders relevant sind. Daher haben Fachverbände (z. B. die Deutsche Gesellschaft für Psychologie: https://lppkjp.de/wp-content/uploads/ 2020/03/EmpfehlungenVideobasierte- 
Psychotherapie_DGPs_IG-E-Health_ 20200326.pdf. Zugegriffen: 01.11.2020), aber auch einzelne Experten (z. B. Kühne und Hintenberger 2020) sehr zeitnah nach den neuen gesetzlichen Regelungen Handzettel oder Publikationen zur Fernbehandlung zusammengestellt, die die wichtigsten Regeln für die Umsetzung von v. a. Videotelefoniesitzungen vermitteln sollen. Ebenso wurden ad hoc KurzWebinare angeboten, um Psychotherapeuten zumindest basale Skills zu vermitteln (z. B. das Webinar am Institut für Klinische Psychologie und Psychotherapie der Universität Greifswald: https:// psychologie.uni-greifswald.de/43051/ lehrstuehle-ii/klinische-psychologieund-psychotherapie/corona-pandemie/ psychologische-unterstuetzung-fuertherapeutinnen/. Zugegriffen: 01.11. 2020).

Natürlich sind diese Handreichungen und Kurzschulungen wichtig, jedoch nur eine Behelfsmaßnahme, die keine qualifizierten Fort- und Weiterbildungen ersetzt. Inzwischen gab es über den Sommer 2020 Zeit, fundiertere und umfassende Weiterbildungen auszuarbeiten. In Deutschland bietet z.B. der Berufsverband für Psychosoziale Berufe eine hybride Fortbildungsveranstaltung für psychologische Psychotherapeuten im Umfang von 20 Unterrichtseinheiten an (www.dgvt-bv.de/ veranstaltungen/online-therapie-undberatung/. Zugegriffen: 01.11.2020) an. In Österreich hat die Weiterbildungsakademie der Sigmund Freud PrivatUniversität den ersten Universitätslehrgang „online@work“ konzipiert (https://weiterbildungsakademie.sfu. ac.at/de/lehrgaenge/ulg-onlineworkpsychosozial/. Zugegriffen: 01.11.2020), der verschiedenen beratenden Berufsgruppen (Psychotherapeuten, Mediziner, Juristen) in jeweils eigenen Zweigen fundiertes Wissen und die praktische Ausübung für das Arbeiten im Videosetting vermittelt. Dieses Angebot ist eine Kooperation mit der Technischen Universität Nürnberg, deren E-HealthInstitut seit vielen Jahren federführend in der Aus- und Weiterbildung in diesem Bereich ist. Natürlich sind diese Weiterbildungsangebote länderübergreifend buchbar.
Auch wenn wir Psychotherapeuten die Herausforderungen im Sinne unserer Patienten angenommen haben, auch ohne Vorerfahrungen und profundes Wissen um die Besonderheiten des Onlinesettings unsere Patienten in diesem neuen Setting Coronakrise-bedingt behandelt $\mathrm{zu}$ haben, so ist notwendig, das videobasierte Behandlungssetting $\mathrm{zu}$ professionalisieren. Das heißt, dass Onlinetherapie zum einen ein integraler Bestandteil der Psychotherapieausbildung wird, zum anderen aber auch eine Weiterqualifizierung der Psychotherapeuten gesetzlich installiert wird, um auch hier qualitätssichernd psychotherapeutische Dienstleistung anbieten zu können. Denn Befragungen zeigen, dass Unsicherheiten bestehen: In der Befragung der Deutschen Psychotherapeutenvereinigung (2020) schätzten $59 \%$ der teilnehmenden Psychotherapeuten die Wirksamkeit der videobasierten Behandlung schlechter ein, im Vergleich zur „Face-to-face“-Behandlung. Von den Befragten wandten $75 \%$ diese an, d.h., auch wenn das Videosetting nicht als Goldstandard gesehen wird. Diese Dissonanz zwischen der coronabedingten Notwendigkeit, im Videosetting zu behandeln, und dieses gleichzeitig als weniger effektiv zu erleben, kann belastend sein, wobei Weiterbildungen durch Kenntnisse in Besonderheiten des Settings, aber auch behandlungstechnische Reflexion einen Ausweg bieten. In Weiterbildungen können ebensolche Fragen (z. B. Wie können die jeweiligen Methoden eines Therapieverfahrens an das Videosetting adaptiert werden?) und Fragen bezüglich der Kriterien für die Behandlungsplanung im digitalen Setting geklärt werden (ausführlich: Eichenberg 2020). Dabei scheint zentral, dass auch Selbsterfahrungsanteile integriert werden, da bisherige Fortbildungen eher auf technische und rechtliche Aspekte fokussierten (s. oben). Schuster et al. (2020) kommen in einer Studie zu dem Ergebnis, dass digitale Therapieoptionen therapeutenseits eher dann positiv bewertet werden, wenn persönliche Erfahrungen damit vorhanden sind. Das heißt, dass Fortbildungsangebote demnach so konzipiert sein müssten, dass Therapeuten die Gelegenheit erhalten, digitale Therapieoptionen aktiv ausprobieren, prüfen und bewerten zu können.

\section{Fazit für die Praxis}

- Die Erfahrungen der Psychotherapeuten mit Onlinepsychotherapie in Zeiten der Coronapandemie sind weitgehend positiv, auch wenn dem digitalen Setting im Vergleich zum traditionellen Setting geringere Wirksamkeit zugeschrieben wird.

- Besondere Vorbehalte bestehen in der Onlinebehandlung von Kindern und Jugendlichen, obwohl auch hier fundierte Konzepte für die (Weiter)Behandlung im Onlinesetting vorhanden sind.

- Dies verdeutlicht die Notwendigkeit umfassender sowie obligatorisch in Aus- und Fortbildungskontexten verankerter Schulungen, um den Berufsstand der Psychotherapeuten fundiert im Bereich Onlinepsychotherapie zu qualifizieren.

- Gleichzeitig wäre es hilfreich, wenn Therapeuten bei ihren Patienten bereits in der Anamnese Aspekte der digitalen Mediennutzung und -präferenzen erheben, um informiert zu sein, welche Onlineoptionen in pandemischen Krisenzeiten gewünscht und möglich sind. Eine eigene Umfrage zeigte, dass dieses Thema in der Therapie bisher wenig Berücksichtigung findet (Eichenberg et al. im Druck c).

\section{Korrespondenzadresse}

Univ.-Prof. Dr. phil. habil.

Christiane Eichenberg

Institut für Psychosomatik, Fakultät für Medizin, Sigmund Freud PrivatUniversität Wien Freudplatz 3, 1020 Wien, Österreich eichenberg@sfu.ac.at

\section{Einhaltung ethischer Richtlinien}

Interessenkonflikt. C. Eichenberg gibt an, dass kein Interessenkonflikt besteht.

Für diesen Beitrag wurden von der Autorin keine Studien an Menschen oder Tieren durchgeführt. Für die aufgeführten Studien gelten die jeweils dort angegebenen ethischen Richtlinien. 


\section{Literatur}

Andersson G, Cuijpers P, Carlbring P et al (2014) Guided Internet-based vs. face-to-face cognitive behavior therapy for psychiatric and somatic disorders: a systematic review and metaanalysis. World Psychiatry 13(3):288-295

Bobo E, Lin L, Purper-Quakil D et al (2020) How do children and adolescents with Attention Deficit Hyperactivity Disorder (ADHD) experience lockdown during the COVID-19 outbreak? Encephale. https://doi.org/10.1016/j.encep. 2020.05.011

Deutsche Psychotherapeutenvereinigung (2020) Umfrage Psychotheapeutische Videobehandlung. https://www.deutschepsychotherapeutenverei nigung.de/index.php?elD $=$ dumpFile \& $t=f \& f=$ 11152\&token $=8$ efba22d7afdbd29ab5f0a824eb 29c7d2aa94b9c.Zugegriffen:25. Okt. 2020

Eichenberg C (2020) Online-Psychotherapie in Zeiten der Corona-Krise. In: Bering R, Eichenberg C (Hrsg) Die Psyche in Zeiten der CoronaKrise. Herausforderungen und Lösungsansätze für Psychotherapeuten und soziale Helfer. KlettCotta, Stuttgart, S69-82

Eichenberg C, Hübner L (2018) Psychoanalyse via Internet: Ein Überblick zum aktuellen Stand der Diskussion um Möglichkeiten und Grenzen. Psychotherapeut 63(4):283-290. https://doi. org/10.1007/s00278-018-0294-0

Eichenberg C, Hübner L (2020) Therapeutische Beziehung im Zeitalter digitaler Medien: Perspektiven und Ergebnisse aus Verhaltenstherapie und psychodynamischer Psychotherapie. In: Merz A, Wildeisen B (Hrsg) Liebes Leben in der Psychiatrie. BoD, Norderstedt, S137-167

Eichenberg C, Kühne S (2014) Einführung Onlineberatung und -therapie. In: Grundlagen, Interventionen und Effekte der Internetnutzung. UTB, München

Eichenberg C, Schott M (2017) Serious games for psychotherapy: a systematic review. Games Health J3:127-135

Eichenberg C, Grabmayer G, Green N (2016a) Acceptance of serious games in psychotherapy: an inquiry into the stance of therapists and patients. Telemed E-health. https://doi.org/10. 1089/tmj.2016.0001

Eichenberg C, Küsel C, Sindelar B (2016b) Computerspiele im Kindes- und Jugendalter: Geschlechtsspezifische Unterschiede in der Präferenz von Spiel-Genres, Spielanforderungen und Spielfiguren und ihre Bedeutung für die Konzeption von Serious Games. Merz Medien Erzieh 6:97-109

Eichenberg C, Grossfurthner M, Andrich J et al (im Druck a) Gleichgültig, verantwortungsbewusst oderüberfordert? EineOnline-Befragungsstudie zumemotionale BefindenjungerErwachsener in der Frühphase des Coronabedingten Lockdowns in Deutschland und Österreich

Eichenberg C, Grossfurthner M, Andrich J et al (im Druck b) Relationship between the implementation of statutory preventive measures and personality traits in the initial stage of corona-related lockdown: a German and Austrian population online survey. Front Psych

Eichenberg C, Piening K, van Loh J (im Druck c). Exploration und Berücksichtigung von Medienproblemen in der Psychotherapie von Erwachsenen: Eine Online-Befragung von Psychotherapeuten

Etzelmüller A, Radkovsky A, Hannig W et al (2018) Patient's experience with blended videoand internet based cognitive behavioural therapy service in routine care. Internet Interv 12:165-175. https://doi.org/10.1016/j.invent. 2018.01.003

Goldberg D, Williams P (1988) A user's guide to the general health questionnaire. NFER-Nelson, Windsor

Humer E, Pieh C, Kuska M et al (2020) Provision of psychotherapy during the COVID-19 pandemic among Czech, German and Slovak psychotherapists. Int J Environ Res Public Health 17(13):4811. https://doi.org/10.3390/ijerph17134811

Kerst A, Zielasek J, Gaebel W (2019) Smartphone applications for depression: a systematic literature review and a survey of health care professionals' attitudes towards their use in clinical practice. Eur Arch Psychiatry Clin Neurosci. https://doi.org/10.1007/s00406-0180974-3

Kühne S, Hintenberger G (2020) Onlineberatung und -therapie in Zeiten der Krise. Ein Überblick. e-beratungsjournal.net, 16, 1, Artikel 3. https://www. e-beratungsjournal.net/wp-content/uploads/ 2020/03/kuehne_hintenberger.pdf. Zugegriffen:01.11.2020

Lemma A, Fonagy P (2013) Feasibility study of a psychodynamic online group intervention for depression and anxiety. Psychoanal Psychol 30(3):367-380

Peñate W, Fumero A (2016) A meta-review of Internet computer-based psychological treatments for anxiety disorders. J Telemed Telecare 22(1):3-11

Pieh C, Budimir S, Probst T (2020) The effect of age, gender, income, work, and physical activity on mental health during coronavirus disease (COVID-19) lockdown in Austria. J Psychosom Res. https://doi.org/10.1016/j.jpsychores.2020. 110186

Pierce M, Hope H, Ford T et al (2020) Mental health before and during the COVID-19 pandemic: a longitudinal probability sample survey of the UK population. Lancet Psychiatry. https://doi. org/10.1016/S2215-0366(20)30308-4

Röhr S, Jung F, Apfelbacher C, Seidler A, RiedelHeller S (2020) Psychosoziale Folgen von Quarantänemaßnahmen bei schwerwiegenden Coronavirus-Ausbrüchen: ein Rapid Review. Psychiatr Prax 47(04):179-189

Schuster R, Topooco N, Keller A et al (2020) Advantages and disadvantages of online and blended therapy: Replication and extension of findings on psychotherapists' appraisals. Internet Interv 21:100326. https://doi.org/10.1016/j.invent. 2020.100326

Simpson SG, Reid CC (2014) Therapeutic alliance in videoconferencing psychotherapy: a review. Aust J Rural Health 22(6):280-299

Sindelar B (2020) Kinder- und Jugendlichenpsychotherapie Online in Zeiten der Corona-Krise. In: Bering R, Eichenberg C (Hrsg) Die Psyche in Zeiten der Corona-Krise. Herausforderungen und Lösungsansätze für Psychotherapeuten und soziale Helfer. Klett-Cotta, Stuttgart, S83-96

Stein J, Röhr S, Luck T et al (2018) Indikationen und Evidenz von international entwickelten OnlineCoaches zur Intervention bei psychischen Erkrankungen - ein Meta-Review. Psychiat Prax 45(01):7-15. https://doi.org/10.1055/s-0043117050

Vigerland S et al (2014) Attitudes towards the use of computerized cognitive behavior therapy (CCBT) with children and adolescents: a survey among Swedish mental health professionals. Interventions. https://doi.org/10.1016/j.invent. 2014.06.002
Wolf M, Zimmer B, Dogs P (2008) Chat- und E-Mail-Brücke: Nachsorge nach stationärer Psychotherapie. In: Bauer S, Kordy H (Hrsg) E-Mental Health. Springer, Berlin, Heidelberg, New York, S219-235

Zurek G (2020) Psychoinformation - Wissen reduziert Befürchtung. In: Bering R, Eichenberg $C$ (Hrsg) Die Psyche in Zeiten der Corona-Krise. Herausforderungen und Lösungsansätze für Psychotherapeuten und soziale Helfer. KlettCotta, Stuttgart, S113-126

Zwerenz R, Becker J, Johansson R et al (2017) Transdiagnostic, psychodynamic web-based self-help intervention following inpatient psychotherapy: results of a feasibility study and randomized controlled trial. JMIR Ment Health 4(4):e41. https://doi.org/10.2196/mental.7889 